\title{
Introduction to the Special Issue on BuildSys'17
}

This special issue of the ACM Transactions on Sensor Networks (TOSN) presents select papers from the 4th ACM International Conference on Systems for Energy-Efficient Built Environments (BuildSys'17), which was held from November 8 and 9, 2017 in Delft, The Netherlands. BuildSys is a highly selective conference that focuses on the design, optimization, implementation, evaluation, and deployment of smart systems, together with the associated modeling, data analytics, and applications for improving the performance and energy-efficiency of built environments, including buildings and critical urban infrastructure. The BuildSys'17 technical program committee chair and several members of the committee served as guest editors for the special issue. Authors of accepted papers at the conference were invited to submit the extended and updated versions of their papers to this special issue. The submitted papers all went through the rigorous TOSN review process, which was expedited by inviting the BuildSys technical program committee members as reviewers. We are proud to present the following set of 15 outstanding articles in this special issue:

- Democratizing Authority in the Built Environment

- Design and Analysis of a Query Processor for Brick

- AutoCalib: Automatic Traffic Camera Calibration at Scale

- Flux: A Platform for Dynamically Reconfigurable Mobile Crowd-sensing

- A Scalable Room Occupancy Prediction with Transferable Time Series Decomposition of CO2 Sensor Data

- A Scalable System for Apportionment and Tracking of Energy Footprints in Commercial Buildings

- Smart Home Occupant Identification via Sensor Fusion Across On-Object Devices

- Optimal Discrete Net-Load Balancing in Smart Grids with High PV Penetration

- Mechanisms and Policies for Controlling Distributed Solar Capacity

- Inverted HVAC: Greenifying Older Buildings, One Room at a Time

- SonicDoor: A Person Identification System Based on Modeling of Shape, Behavior, and Walking Patterns

- Maintenance of Smart Buildings Using Fault Trees

- sTube+: An IoT Communication Sharing Architecture for Smart After-sales Maintenance in Buildings

- A Framework for Privacy-preserving Data Publishing with Enhanced Utility in Cyberphysical Systems

- Designing Green Communication Systems for Smart and Connected Communities via Dynamic Spectrum Access

We appreciate all the contributors who made this special issue possible, including the authors who submitted their articles and the reviewers who provided valuable and timely feedback to ensure the quality of this special issue. We hope this special issue provides new inspirations and opportunities for future research in the field.

Hae Young Noh

Xiaofan (Fred) Jiang

Pei Zhang

2018 Copyright is held by the owner/author(s).

1550-4859/2018/12-ART16

https://doi.org/10.1145/3289594 